\title{
Éditorial / Editorial
}

\section{Les aphorismes de l'éducation thérapeutique}

Un rapport récent de l'inspection générale des affaires sociales portant sur la prise en charge du diabète de type 2 en France, souligne les difficultés et l'inadaptation de l'éducation thérapeutique depuis sa reconnaissance officielle par la loi HPST. Ce rapport critique le formalisme des programmes d'éducation thérapeutique validés par les agences régionales de santé (ARS) souvent trop lourds. Il constate que l'éducation thérapeutique touche insuffisamment les populations les plus à risque de complications du diabète que ce soit pour des raisons médicales, sociales, culturelles ou psychologiques.

Le rapport reflète en même temps la grande confusion qui règne aujourd'hui sur la question de l'éducation thérapeutique, et il paraît donc utile de rappeler quelques aphorismes :

1) l'éducation thérapeutique a trois composantes indissociables :

- l'individualisation du traitement et des objectifs,

- l'acquisition par le patient de compétences grâce à une pédagogie active,

- l'aide à la mise en place dans la durée par le patient de ses nouvelles compétences dans sa vie quotidienne.

2) l'éducation thérapeutique nécessite un temps et un lieu dédiés, qui peut aller d'une consultation non prescriptive à un atelier de groupe. Le formalisme des programmes d'éducation thérapeutique validé par les ARS entraîne une rigidité incompatible avec une pratique centrée sur le patient.

3) l'éducation thérapeutique s'est développée depuis 30 ans à l'hôpital public et pour deux raisons : d'une part l'existence d'équipes médicales et paramédicales travaillant ensemble, d'autre part un financement par un budget global. Il ne s'est développé que secondairement dans les centres de santé et les réseaux pour ces deux mêmes raisons.

4) l'éducation thérapeutique pour les pathologies chroniques fréquentes comme le diabète de type 2 , doit comporter deux niveaux :

- Un premier niveau dit de premier recours, simple, réalisé essentiellement par les paramédicaux à la demande des médecins traitants. Les médecins traitants doivent pouvoir travailler avec des infirmières, des diététiciennes sous une forme adaptée (cabinets, maisons pluri-professionnelles de santé, centres de santé, réseaux...). Cette éducation thérapeutique devrait être réalisée au plus près des lieux de vie habituelle du patient.

- Un niveau spécialisé (plus souvent hospitalier) est nécessaire pour les pathologies rares, les traitements complexes, les situations d'échec de la prise en charge du niveau de premier recours, c'est-à-dire pour des patients présentant des complications évolutives ou les patients à haut risque de survenue des complications. Elle nécessite une équipe médicale et paramédicale ayant une triple expertise thérapeutique, pédagogique et psychologique.

5) l'hôpital ambulatoire est en ville ! La séparation habituelle entre l'hôpital et la ville n'est pas pertinente quand il s'agit d'une activité ambulatoire, le patient restant moins d'une journée à l'hôpital. En conséquence, les spécialistes hospitaliers médicaux et paramédicaux de l'hôpital devraient pouvoir aller travailler avec leurs confrères de ville quand cela est nécessaire, et inversement les médecins et paramédicaux de ville devraient pouvoir travailler dans les structures hospitalières ambulatoires d'éducation thérapeutique. Plusieurs hôpitaux développent d'ailleurs des structures mixtes hôpital-ville, situées symboliquement à l'entrée de l'hôpital et ouvrant directement sur la ville. Encore faut-il que les professionnels aient le temps et qu'il existe un financement adapté.

Le financement de l'éducation thérapeutique nécessite de dégager, d'après le rapport de l'IGAS, plusieurs centaines de millions d'euros sur les 13 milliards dépensés chaque année pour la prise en charge des patients diabétiques. Cela paraît tout à fait possible si les professionnels, les usagers, et les institutionnels (Haute Autorité de Santé et Sécurité sociale) acceptent de rediscuter le «panier de soins » pris en charge à $100 \%$ : tout ce qui est utile et rien de ce qui est inutile. À efficacité comparable c'est le traitement le moins cher qu'il faut privilégier. Les uns et les autres, y sont-ils prêts ? Ce serait vraiment le changement!

André Grimaldi 